\title{
Physical activity measures in patients with myalgic encephalomyelitis/chronic fatigue syndrome: correlations between peak oxygen consumption, the physical functioning scale of the SF-36 questionnaire, and the number of steps from an activity meter
}

C (Linda) MC van Campen

Stichting CardioZorg, Planetenweg 5, 2132 HN Hoofddorp, NL

Peter C Rowe ( $\triangle$ prowe@jhmi.edu )

Johns Hopkins University School of Medicine https://orcid.org/0000-0002-3586-2677

Freek WA Verheugt

Onze Lieve Vrouwe Gasthuis (OLVG), Oosterpark 9, 1091 AC Amsterdam, NL

\section{Frans C Visser}

Stichting CardioZorg, Planetenweg 5, 2132 HN Hoofddorp, NL

\section{Research}

Keywords: SensewearTM armband, chronic fatigue syndrome, spiro-ergometry, peak VO2, SF 36 questionnaire, myalgic encephalitis

Posted Date: April 8th, 2020

DOI: https://doi.org/10.21203/rs.3.rs-21479/v1

License: (c) (i) This work is licensed under a Creative Commons Attribution 4.0 International License.

Read Full License

Version of Record: A version of this preprint was published at Journal of Translational Medicine on June 8th, 2020. See the published version at https://doi.org/10.1186/s12967-020-02397-7. 


\section{Abstract}

Background most studies to assess effort intolerance in patients with myalgic encephalomyelitis/chronic fatigue syndrome (ME/CFS) have used questionnaires. Few studies have compared questionnaires with objective measures like an actometer or an exercise test. Therefore three measures of physical activity in ME/CFS patients, being the physical functioning scale (PFS) of the SF-36, the number of steps/day (Steps) of an actometer and the \%peak VO 2 of a cardiopulmonary stress test were compared.

Methods female ME/CFS patients were selected from a clinical database if the three types of measurements were available, and the interval between measurements was $\leq$ three months. Data of the three measures were compared by linear regression.

Results in 99 female patients the three different measures were linearly, significantly and positively correlated (PFS vs Steps, PFS vs \%peak VO 2 and Steps vs \%peak VO 2 : all P<0.001). Subgroup analysis showed that the relations between the three measures were not different in patients with versus without fibromyalgia and with versus without a maximal exercise effort ( $R E R \geq 1.1)$. In 20 patients re-evaluated for symptom worsening, the mean of all three measures was significantly lower $(P<0.0001)$, strengthening the observation of the relations between them. Despite the close correlation a large variation was observed between the three measures in individual patients.

Conclusions given the large variation in ME/CFS patients, the use of only one type of measurement is inadequate. Integrating the three modalities may be useful for patient care by detecting overt discrepancies and may aid study designs aimed at improving exercise capacity.

\section{Background}

Myalgic Encephalomyelitis/Chronic Fatigue Syndrome (ME/CFS) is a serious and potentially disabling chronic disease [1-3]. One of the defining characteristics of patients with ME/CFS is effort intolerance and a prolonged recovery after increased activity $[4,5]$. The pathophysiology of the exercise intolerance is not known but involves both metabolic abnormalities of skeletal muscles as well as central nervous system abnormalities [5-11]. Thus, by definition, ME/CFS involves some degree of disability, defined as "any restriction or lack (resulting from an impairment) of ability to perform an activity in the manner or within the range considered normal for a human being" [12].

Disability in ME/CFS is multi-factorial, with social, physical, mental, training and labour dimensions. The majority of ME/CFS studies have employed questionnaires to describe the extent of disability. One of the commonly used questionnaires is the SF-36 and especially the physical functioning scale of this questionnaire [13-20].

Objective measures of functional status and wellbeing have the potential to augment and improve the validity of questionnaire measures. Exercise intolerance can be quantified by using an activity meter [21] and by measuring peak oxygen consumption on a cardiopulmonary stress test (CPET) [22-33]. We are 
not aware of studies measuring how well questionnaires and these two objective measurements correlate in ME/CFS populations. Therefore, the aim of this study was to correlate peak oxygen consumption with the number of steps on the Sensewear ${ }^{\mathrm{TM}}$ armband and the physical functioning scale of the SF-36 in ME/CFS patients. We elected to answer this question by studying female ME/CFS patients, because of differences in peak oxygen consumption between males and females, and possible gender differences in the clinical phenotype of the disease [34-36]

\section{Methods}

\section{Participants}

This was a retrospective study of patients referred between 2012 and 2019 to the Stichting CardioZorg, a cardiology clinic that specializes in the assessment and treatment of those with CFS and ME. All eligible participants had been referred by their general practitioners for the diagnosis of ME/CFS. Patients underwent a detailed clinical history, physical examination, laboratory analysis, ECG and echocardiography. Based on their symptoms the diagnosis of chronic fatigue syndrome (CFS) according to the Fukuda Criteria [1] and myalgic encephalomyelitis (ME) according to the international ME criteria [2] was established. In all patients alternative diagnoses which could explain the fatigue and other symptoms were ruled out. We excluded patients with a body mass index of $\geq 50$ because the normal reference values for the CPET [37] were based on female healthy volunteers with a BMI up to 50. The diagnosis of fibromyalgia was based on either the previous diagnosis of a rheumatologist or based on the American College of Rheumatology questionnaire 2010 [38].

A subset of patients completed the SF-36 questionnaire, wore a Sensewear ${ }^{\mathrm{TM}}$ armband for five days and underwent cardiopulmonary exercise testing. These tests were mainly done to demonstrate the degree of disability because of conflicts with the social security administration.

From among those who had undergone all three tests, to minimize between measures variability in there functional status, we selected for study those in whom the interval between the SF-36 questionnaire, the Sensewear ${ }^{\text {TM }}$ measurements and the cardiopulmonary stress test was less than 3 months. In addition, patients who were re-evaluated because of worsening of symptoms were analyzed separately.

The study was carried out in accordance with the Declaration of Helsinki. The use of clinical data for descriptive studies was approved by the ethics committee of the Slotervaart Hospital, the Netherlands (P1450). All patients give informed consent to analyze their data.

\section{Study measures}

Cardiopulmonary exercise testing (CPET): patients underwent a symptom-limited exercise test on a cycle ergometer (Excalibur, Lode, Groningen, The Netherlands) according to a previously described protocol [22]. A RAMP workload protocol was used varying between 10-30 Watt/min increases, depending on sex, age and expected exercise intolerance. Oxygen consumption $\left(\mathrm{VO}_{2}\right)$, carbon dioxide release $\left(\mathrm{VCO}_{2}\right)$ and 
oxygen saturation were continuously measured (Cortex, Procare, The Netherlands), and displayed on screen using Metasoft software (Cortex, Biophysic Gmbh, Germany). An ECG was continuously recorded and blood pressures were measured continuously using the Nexfin device (BMEYE, Amsterdam, The Netherlands) [39]. The test was supervised by an experienced cardiologist. Patients were encouraged by standard phrases each minute to perform maximally to the point of exhaustion. The mean of the $\mathrm{VO}_{2}$ measurements of the last 15 seconds before ending the exercise (peak $\mathrm{VO}_{2}$ ) was taken, and expressed as a percentage of the normal values of a population study: \%peak $\mathrm{VO}_{2}$ [37]. We assessed the mean respiratory exchange ratio (RER; $\mathrm{VCO}_{2} / \mathrm{VO}_{2}$ ) of the last 15 seconds to determine the influence of this measure of maximal effort on the results. Immediately after the test the attending cardiologist noted the primary reason for termination the exercise and judged whether motivation and efforts during exercise were optimal for the individual patient.

Sensewear ${ }^{\mathrm{TM}}$ armband (BodyMedia, Pittsburgh, PA, USA) measurements: Patients wore the armband for approximately 4 days and were advised to remove the armband only during showering or bathing. From the armband data, the number of steps was recorded and normalized to $24 \mathrm{hrs}$. The coefficient of variation of the number of steps per day was calculated. After wearing the armband patients were asked if the 4 days were "average" days. If there was a gross over- or under-activity, patients were excluded from the analysis. Furthermore, patients who wore the armband less than 23 hrs. per day were excluded.

SF-36 physical functioning subscale: this subscale of the SF-36 asks whether the respondents health limits activities performed during a typical day a lot, a little, or not at all. The Dutch version of the SF-36 for physical functioning [40] was used. The scores of the 10 items of the questionnaire regarding physical activity were transformed into a scale ranging from $0-100 \%$.

\section{Data analysis}

Data were analyzed using the statistical package of Graphpad Prism version 6.05 (Graphpad software, La Jolla, California, USA). All continuous data were tested for normal distribution using the D'AgostinoPearson omnibus normality test, and presented as mean (SD) or as median with the IQR, where appropriate.

Linear regression was performed to assess the relation between measurements (physical function subscale of SF-36, number of steps, and CPET percentage peak $\mathrm{VO}_{2}$ ).

A paired $T$ test was performed on patients who underwent re-evaluation because of worsening symptoms. Because of the multiple comparisons a more conservative $\mathrm{P}$ value was chosen in which a $\mathrm{P}<$ 0.01 was considered significantly different.

\section{Results}

Between October 2012 and January 2019, 844 patients visited the outpatient clinic of the Stichting CardioZorg and were diagnosed ME/CFS. In 197 female patients the three studies were available. Of 
these, 110 had all three studies performed within 3 months. Of the 110,4 patients were excluded because of gross under- or over-activity compared to their average daily activity, 1 because of an insufficient armband wear time, and 1 because of recording artifacts. Two patients were excluded because of motion artifacts (both had a ride of more than $1 \mathrm{hr}$ on a motorbike/scooter, during which the Sensewear ${ }^{\text {TM }}$ device reflected vigorous activity). Three patients experienced an allergic skin reaction to the armband that disrupted the recording. Thus, 99 patients were analyzed. All electrocardiograms were normal; the echocardiograms were either normal or showed minor abnormalities like mild valvular regurgitation and mild left atrial enlargement. No left ventricular dysfunction was present. In none of the patients moderate to severe COPD was present. Nineteen patients were re-evaluated because of symptom worsening and underwent repeat CPET, activity tracking, and completion of the SF-36 questionnaire.

Table 1 shows the characteristics of the 99 female study participants with ME/CFS. The interval between the CPET and SF questionnaire was less than 1 week in all participants. The median interval between the SF-36 questionnaire and Sensewear ${ }^{\text {TM }}$ measurements was 2 months (IRQ 1-3 months) and the median interval between the CPET and Sensewear ${ }^{\text {TM }}$ was 1 month (IRQ: 1-2 months). Of the Sensewear ${ }^{\mathrm{TM}}$ measurements the between days variation of the number of steps of an individual patient was expressed as the coefficient of variation. The median coefficient of variation of the number of steps in the total population was 7\% (IQR: 3-16\%). CPET data: using a RER $>=1.1$ as a criterion for a maximal exercise(41), 53 patients (54\%) reached a RER above 1.1. The primary reason for termination of the exercise was muscle strength exhaustion or muscle pain in 86 patients (87\%), shortness of breath in 9 patients $(9 \%)$ and other reasons in 4 patients $(4 \%)$. The attendant cardiologist judged patient motivation and efforts to be maximal in all patients. 
Table 1

Demographic features and main activity measures*

\begin{tabular}{|c|c|}
\hline Number of patients & 99 \\
\hline Age in years & $41(11)$ \\
\hline Fibromyalgia (number/percentage) & $58(59 \%)$ \\
\hline $\mathrm{BMI}$ in $\mathrm{kg} / \mathrm{m}^{2}$ & $24.4(5.2)$ \\
\hline Disease duration in years (median/IQR) & $12(6-7)$ \\
\hline SBP at rest in $\mathrm{mmHg}$ & $125(12)$ \\
\hline DBP at rest in $\mathrm{mmHg}$ & $78(8)$ \\
\hline Heart rate at rest in bpm & $80(13)$ \\
\hline Number of steps/day (median/IQR) & $4683(3269-7399)$ \\
\hline SF-36 Physical Activity scale & $46(21)$ \\
\hline$\%$ peak VO & $76(18)$ \\
\hline RER & $1.11(0.11)$ \\
\hline RER $\geq 1.10$ (number/percentage) & $53(54 \%)$ \\
\hline
\end{tabular}

Figure 1 shows the correlations and the $95 \%$ prediction intervals between the \%peak $\mathrm{VO}_{2}$, the $\mathrm{SF}-36$ physical functioning scale, and the number of steps per day. All relations were highly significant (all $p<$ 0.0001).

To evaluate whether the association between measures was affected by conventional definitions of effort expended, as measured by the RER, a subgroup analysis was done in ME/CFS patients with and without a RER $\geq 1.1$. To evaluate the influence of muscle pain on exercise termination we also compared ME/CFS patients with and without fibromyalgia. The RER in ME/CFS patients with fibromyalgia $(n=58)$ was significantly lower $(1.07(0.11))$ compared to ME/CFS patients without fibromyalgia $(n=41)$ (RER 1.17 (0.10)), $\mathrm{P}<0.0001$. 
Table 2

A Subgroup analysis of ME/CFS patients with and without a RER $\geq 1.1$

\begin{tabular}{|llll|}
\hline & RER $\geq 1.1$ & RER $<1.1$ & P-value \\
\hline Number/percentage & $53(54 \%)$ & $46(46 \%)$ & \\
\hline Number of steps/day (median/IQR) & $4683(3350-7932)$ & $4779(3352-7279)$ & 0.30 \\
\hline SF-36 Physical Activity & $49(20)$ & $38(22)$ & 0.03 \\
\hline$\%$ peak VO & $78(18)$ & $71(18)$ & 0.09 \\
\hline
\end{tabular}

Table 2

B Subgroup analysis of ME/CFS patients with and without fibromyalgia

\begin{tabular}{|llll|}
\hline & Fibromyalgia + & Fibromyalgia - & P-value \\
\hline Number/percentage & $58(58 \%)$ & $41(41 \%)$ & \\
\hline Number of steps/day (median/IQR) & $4527(3264-6235)$ & $6401(3531-8123)$ & 0.14 \\
\hline SF-36 Physical Activity & $42(19)$ & $52(22)$ & 0.02 \\
\hline$\%$ peak $\mathrm{VO}_{2}$ & $74(19)$ & $80(16)$ & 0.08 \\
\hline *All values are mean (SD) unless otherwise noted. For abbreviations see Table 1. & \\
\hline
\end{tabular}

Table 2A shows that there is no significant difference between patients with a peak RER $\geq 1.1$ vs patients with a peak RER $<1.1$ in the number of steps/day, the physical functioning scale of the SF-36 and the $\%$ peak $\mathrm{VO}_{2}$. Also there was no difference in these parameters in patients with and without fibromyalgia as shown in Table 2B.

Figures 2 and 3 show the correlations and the 95\% prediction intervals between the \%peak $\mathrm{VO}_{2}$, the SF-36 physical functioning scale, and the number of steps per day in patients with and without a RER $>1.1$ (Fig. 2) and in patients with and without fibromyalgia (Fig. 3). In patients with a RER $<=1.1$ the relation between the \%peak $\mathrm{VO}_{2}$ and the number of steps per day was significant at the level of $\mathrm{P}<0.005$, all other relations in patients with and without a RER $>1.1$ were significant at the level of $P<0.0001$. There were no significant differences between the regression lines between patients with and without a RER $>1.1$. In patients without fibromyalgia the relation between the \%peak $\mathrm{VO}_{2}$ and the SF-36 physical functioning scale and between the \%peak $\mathrm{VO}_{2}$ and the number of steps per day were significant at the level of $\mathrm{P}<$ 0.005. All other relations in patients with and without fibromyalgia were significant at the level of $P<$ 0.0001 . There were no significant differences between the regression lines in patients with and without fibromyalgia. 
Figure 4 shows the \% of normal peak oxygen consumption, the physical functioning scale of the SF-36, and the number of steps/day in 20 patients undergoing re-evaluation because of worsening of symptoms. Re-evaluation was performed a median of 19 months after the initial visit (IRQ: 14-24 months). All three types of measurements showed a significant deterioration for the number of steps/day, for the physical functioning scale of the SF-36 and for the \%peak $\mathrm{VO}_{2}$ in comparison to the initial evaluation (all $p<0.0001$ ).

\section{Discussion}

The main finding of this study was that all disability measurements were highly significantly correlated: the physical functioning scale of the SF-36, the number of steps on an activity meter, and the percentage peak $\mathrm{VO}_{2}$ relative to a reference value of healthy controls. Few studies have been performed to validate disability measurements in ME/CFS patients, especially with the use of more objective measures other than history taking. To the best of our knowledge this is the first study to have measured all three in determining activity levels in ME/CFS patients.

In comparison to values reported in other ME/CFS populations, our study participants had similar steps per day [21], and \%peak $\mathrm{VO}_{2}$ values [22, 27, 30]. The reported median or mean physical functioning scale of the SF-36 questionnaire vary widely between studies ranging between 17 and 59\% [13-18]. This large variation is probably due to patient selection. Inclusion of more severely affected patients (with lower physical functioning scales) reduces the mean of the data. This is shown in the study of Pendergrast et al. [18] where the housebound patients had a mean physical functioning scale of $17 \%$ while the nonhousebound patients had a score of $42 \%$. In the present study the mean physical functioning scale was $46 \%$, with a range between 0 and $95 \%$, indicating a large variation in ME/CSF severity. This strengthens the generalizability of the observed relations between $\mathrm{VO}_{2}$ and activity measurements and the physical function scale.

All three types of measurements in this study are related to the activity level of patients. Activity in women is partially determined by age, race, menopausal status, educational level, body mass index, depressive symptoms, smoking, chronic medical conditions, and pain [42]. The peak $\mathrm{VO}_{2}$ is influenced by genetics, gender, age, training status, exercise mode, bedrest, altitude, body composition, medication, the capacity of the respiratory and circulatory systems to take up and transport oxygen, and the capacity of the working muscles to receive and use oxygen. In ME/CFS patients the degree of fatigue/exhaustion, post-exertional malaise, underlying metabolic abnormalities, fibromyalgic pain, kinesiophobia and the use of medication may further influence physical activities.

A large number of studies have examined the validity of the SF-36 questionnaire, showing that the physical functioning scale discriminates between various diseases and healthy controls [43]. In ME/CFS patients Jason et al. reviewed the ability of the different subscales of the SF-36 questionnaire to discriminate CFS patients from healthy controls for the goal of operationalizing the measurement of "substantial reductions in previous levels of occupational, educational, social, or personal activities" [44]. 
The authors found that the physical functioning subscale was slightly less than optimal to discriminate between patients and healthy controls, using an area-under-the-curve (AUC) cut-off value of $>0.90$ for optimal discrimination. In the community-based sample the AUC of the physical functioning scale was 0.84 and in the tertiary care sample 0.87 . However, another study found an AUC for assessing substantial reductions of the physical functioning scale of 0.91 [45], suggesting that the use of the physical functioning score is valid with an acceptable sensitivity and specificity.

Several studies have shown a decreased peak $\mathrm{VO}_{2}$ in ME/CFS patients [46]. However, only one study determined the relation between the peak $\mathrm{VO}_{2}$ and accelerometer data in female ME/CFS patients: higher peak $\mathrm{VO}_{2}$ values were related to a higher physical activity time, physical activity energy expenditure, and a mean energy expenditure [47]. In the present study a significant linear relation was found between the peak $\mathrm{VO}_{2}$ and the number of steps as assessed by the Sensewear ${ }^{\mathrm{TM}}$ meter. Given the above mentioned large number of influencing factors on peak $\mathrm{VO}_{2}$ and physical activity in combination with the variation of steps on consecutive days, it is not surprising that, although a very significant relation between peak $\mathrm{VO}_{2}$ and the number of steps exists, the prediction intervals are wide.

There are also limited data available on the relation between peak $\mathrm{VO}_{2}$ and activity questionnaires. One study found a moderate association between exercise capacity and, using the Chronic Fatigue Syndrome Activities and Participation Questionnaire, activity limitations/participation restrictions in patients with $\mathrm{ME} / \mathrm{CFS}$ [48]. Our data on the relation between peak $\mathrm{VO}_{2}$ and the physical function scale are consistent with their observations.

With respect to the relation between the physical functioning score and the activity meter, a previous study failed to demonstrate a difference in activity, as measured by an actometer, between two CFS groups, one with a mean physical functioning score of $54 \%$ and another with a mean score of $33 \%$ [49]. One case study noted a discrepancy between the psychometrically assessed improvement in function after graded exercise therapy versus the decrease in steps after therapy [50]. In the present study, we found a significant relation between the physical function scale and the number of steps; this was the strongest correlation in our study. The close correlation is not unexpected in ME/CFS patients as a previous study showed that ME/CFS patients are more aware of their daily physical activities compared to healthy controls [51]. Nevertheless, there is considerable variation between individual patients at specific levels of the physical functioning scale. For example, at the level of $30 \%$ of the physical functioning scale, the number of steps of individual patients varied between 1558 and 4266 . At a physical functioning scale of $60 \%$, the number of steps varied between 6277 and 9641 , reflecting quite different function. The same holds true for the peak $\mathrm{VO}_{2}$ versus the number of steps taken. For a peak $\mathrm{VO}_{2}$ between 50 and $60 \%$ of normal the number of steps of patients ranged between 1135 and 4683 .

Although the physical functioning scale is adequate to make a distinction between a group of diseased and non-diseased individuals, the physical functioning scale is less useful to describe the degree of disability in individual patients, given the variation of the number of steps for a certain value of the 
physical functioning scale. An integrated approach of more than one type of measurement is therefore needed for the purposes of research study outcomes, disability determination and individual patient management.

Our observation of the relation between the peak $\mathrm{VO}_{2}$, the number of steps and the physical functioning scale is further strengthened by the observation that 20 patients who were re-evaluated because of worsening of symptoms had a significant reduction in peak $\mathrm{VO}_{2}$, the number of steps and the physical functioning scale (Fig. 2). It is interesting to note that 1 of the 20 patients who presented with worsening of symptoms actually had an improvement in the number of steps/day and an unchanged physical function scale (individual data not shown). This stresses the notion that symptomatology needs to be confirmed by objective measurements.

It can be argued that the peak $\mathrm{VO}_{2}$ is the limit for a certain amount of activity: the lower the peak $\mathrm{VO}_{2}$ the less activity can performed or steps taken. Ideally, nomograms of the relation between peak $\mathrm{VO}_{2}$, number of steps per day and the physical functioning scale should be available for ME/CFS patients, allowing clinicians to determine whether patients have gross over- or under-performance (in terms of number of steps) and explore the reasons for this over- and underperformance. Similarly, an imbalance between the physical function scale and the number of steps could be explored. This could also be beneficial for patient activity management. However, from the present data the construction of nomograms is not possible and future studies are needed.

Our data highlight a discrepancy between recommendations for CPET. In studies measuring \%peak $\mathrm{VO}_{2}$, guidelines have advocated using the RER for assigning a test as maximal effort using an RER > = 1.1 [52]. In our subgroup analysis, $54 \%$ of the patients reached a peak RER $>=1.1$ while $46 \%$ reached a peak RER < 1.1. Despite this difference (by definition $\mathrm{P}<0.0001$ ), the \%peak $\mathrm{VO}_{2}$, the number of steps/day and the physical functioning scale of the SF-36 were not different between patients reaching a peak RER $>=1.1$ and patients with a RER $<1.1$. Moreover, the relations between the three measurements: \%peak $\mathrm{VO}_{2}$, number of steps per day and the physical functioning scale were not different between patients with and without a RER $>=1.1$. In ME/CFS patients a number of studies have shown that metabolic skeletal muscle abnormalities are present $[5,53,54]$. These skeletal muscles abnormalities may limit the maximal performance with RER values above 1.1. As Mezzani stated: "It must be borne in mind, however, that patients with severely impaired exercise tolerance can attain skeletal muscle strength exhaustion even earlier than central hemodynamic and ventilatory factors become limiting, interrupting exercise at peak respiratory exchange ratio values even lower than 1.00" [41]. Our data therefore suggest that an RER $<1.1$ should not be used as an exclusion criterion for future studies of exercise performance in ME/CFS patients. Further support for this position comes from examining the fibromyalgia subgroup. In the present study $59 \%$ of the ME/CFS patients had a concomitant diagnosis of fibromyalgia. In all these patients, the reason for exercise termination was leg muscle pain in combination with leg muscle strength exhaustion. In these fibromyalgia patients the RER was significantly lower than in the ME/CFS patients without fibromyalgia. The low RER in fibromyalgia patients can be explained by the earlier exercise 
termination because of muscle pain. The \%peak $\mathrm{VO}_{2}$, the number of steps and the physical functioning scale were not different between patients with and without fibromyalgia. This observation again argues against the use of a low RER as exclusion criterion.

\section{Limitations}

This was a retrospective analysis taking data from patients with a maximum interval of 3 month between the 3 different measurements. We made the assumption that the clinical course would be stable over this period of time, but the retrospective nature of the study did not allow us to confirm this. A prospective study would be needed to evaluate the variability in measurements over time. Because of the limited number of patients, we did not correct for all the confounding factors as mentioned above for the three measurements. The Sensewear ${ }^{\text {TM }}$ activity meter is not available anymore, but the present commercial actographs and smart watches have step measurements included. Only patients who were evaluated because of worsening of symptoms were re-analysed. We have no repeat data on patients who were stable or who improved.

\section{Conclusion}

Disability grading or activity assessment is most frequently done by questionnaires like the physical functioning scale of the SF-36. The assessment can be augmented by adding more objective measures as number of steps on an activity meter and/or by adding peak $\mathrm{VO}_{2}$ data. The relation between the physical functioning scale and the number of steps, and the relation between activity (steps), perceived activity (physical functioning scale) and the maximum attainable activity $\left(\right.$ peak $\mathrm{VO}_{2}$ ) may give insight into a possible over- or underestimation of the perceived activity and over- or under-performance of physical activities. Whether a better "energy management" leads to stabilization or improvement over time of patients, needs to be studied. Finally, the presented standard deviations may aid the design of outcome studies.

\section{Abbreviations}

$\mathrm{BMI}$

body mass index

CFS

chronic fatigue syndrome

CPET

cardiopulmonary exercise test

ME

myalgic encephalitis

VO2

oxygen consumption

vCO2 
carbon dioxide release

\%peak VO2

The VO2 at peak exercise normalized to a population reference value

\section{Declarations}

Ethics approval and consent to participate

The study was carried out in accordance with the Declaration of Helsinki. The use of clinical data for descriptive studies was approved by the ethics committee of the Slotervaart Hospital, the Netherlands (P1450). All patients give informed consent to analyze their data.

Consent for publication

Not applicable

Availability of data and materials

The datasets analysed in the current study are available from the corresponding author on reasonable request.

Competing interests

The authors declare that they have no competing interests.

Authors 'contributions

CMCVC, PCR, and FCV conceived the study, CMCVC and FCV collected the data, CMCVC performed the primary data analysis and FCV, FWAV and PCR performed secondary data analyses. All authors were involved in the drafting and review of the manuscript.

Funding:

This work was not funded.

Acknowledgement statement:

Dr Rowe is supported by the Sunshine Natural Wellbeing Foundation Professorship.

\section{References}

1. Fukuda K, Straus SE, Hickie I, Sharpe MC, Dobbins JG, Komaroff A, et al. The chronic fatigue syndrome: a comprehensive approach to its definition and study. International Chronic Fatigue Syndrome Study Group. Ann Intern Med. 1994;121:953-9. 
2. Carruthers BM, van de Sande MI, DE Meirleir KL, Klimas NG, Broderick G, Mitchell T, et al. Myalgic encephalomyelitis: International Consensus Criteria. J Intern Med. 2011;270:327-38.

3. Clayton EW. Beyond myalgic encephalomyelitis/chronic fatigue syndrome: an IOM report on redefining an illness. JAMA. 2015;313:1101-2.

4. Paul L, Wood L, Behan WM, Maclaren WM. Demonstration of delayed recovery from fatiguing exercise in chronic fatigue syndrome. Eur J Neurol. 1999;6:63-9.

5. Jones DE, Hollingsworth KG, Taylor R, Blamire AM, Newton JL. Abnormalities in pH handling by peripheral muscle and potential regulation by the autonomic nervous system in chronic fatigue syndrome. J Intern Med. 2010;267:394-401.

6. Fulle S, Pietrangelo T, Mancinelli R, Saggini R, Fano G. Specific correlations between muscle oxidative stress and chronic fatigue syndrome: a working hypothesis. J Muscle Res Cell Motil. 2007;28:355-62.

7. McCully KK, Malucelli E, lotti S. Increase of free Mg2 + in the skeletal muscle of chronic fatigue syndrome patients. Dyn Med. 2006;5:1.

8. Wong R, Lopaschuk G, Zhu G, Walker D, Catellier D, Burton D, et al. Skeletal muscle metabolism in the chronic fatigue syndrome. In vivo assessment by 31P nuclear magnetic resonance spectroscopy. Chest. 1992;102:1716-22.

9. McCully KK, Smith S, Rajaei S, Leigh JS Jr, Natelson BH. Blood flow and muscle metabolism in chronic fatigue syndrome. Clin Sci(Lond). 2003;104:641-7.

10. Gur A, Oktayoglu P. Central nervous system abnormalities in fibromyalgia and chronic fatigue syndrome: new concepts in treatment. Curr Pharm Des. 2008;14:1274-94.

11. Siemionow V, Fang Y, Calabrese L, Sahgal V, Yue GH. Altered central nervous system signal during motor performance in chronic fatigue syndrome. Clin Neurophysiol. 2004;115:2372-81.

12. WHO. International Classification of Functioning, Disability and Health (ICF). Geneva: World Health Organization; 2001.

13. Nacul LC, Lacerda EM, Campion P, Pheby D, Drachler ML, Leite JC, et al. The functional status and well being of people with myalgic encephalomyelitis/chronic fatigue syndrome and their carers. BMC Public Health. 2011;11:402.

14. Murdock KW, Wang XS, Shi Q, Cleeland CS, Fagundes CP, Vernon SD. The utility of patient-reported outcome measures among patients with myalgic encephalomyelitis/chronic fatigue syndrome. Qual Life Res. 2017;26:913-21.

15. Kingdon CC, Bowman EW, Curran H, Nacul L, Lacerda EM. Functional status and well-being in people with myalgic encephalomyelitis/chronic fatigue syndrome compared with people with multiple sclerosis and healthy controls. Pharmacoecon Open. 2018.

16. Hardt J, Buchwald D, Wilks D, Sharpe M, Nix WA, Egle UT. Health-related quality of life in patients with chronic fatigue syndrome: an international study. J Psychosom Res. 2001;51:431-4. 
17. Johnston SC, Brenu EW, Hardcastle SL, Huth TK, Staines DR, Marshall-Gradisnik SM. A comparison of health status in patients meeting alternative definitions for chronic fatigue syndrome/myalgic encephalomyelitis. Health Qual Life Outcomes. 2014;12:64.

18. Pendergrast T, Brown A, Sunnquist M, Jantke R, Newton JL, Strand EB, et al. Housebound versus nonhousebound patients with myalgic encephalomyelitis and chronic fatigue syndrome. Chronic Illness. 2016;12:292-307.

19. Fenouillet E, Vigouroux A, Steinberg JG, Chagvardieff A, Retornaz F, Guieu R, et al. Association of biomarkers with health-related quality of life and history of stressors in myalgic encephalomyelitis/chronic fatigue syndrome patients. J Transl Med. 2016;14:251.

20. Buchwald D, Pearlman T, Umali J, Schmaling K, Katon W. Functional status in patients with chronic fatigue syndrome, other fatiguing illnesses, and healthy individuals. Am J Med. 1996;101:364-70.

21. Asprusten TT, Sulheim D, Fagermoen E, Winger A, Skovlund E, Wyller VB. Systemic exertion intolerance disease diagnostic criteria applied on an adolescent chronic fatigue syndrome cohort: evaluation of subgroup differences and prognostic utility. BMJ Paediatr Open. 2018;2:e000233.

22. Vermeulen RC, Vermeulen van Eck IW. Decreased oxygen extraction during cardiopulmonary exercise test in patients with chronic fatigue syndrome. J Transl Med. 2014;12:20.

23. De Becker P, Roeykens J, Reynders M, McGregor N, De Meirleir K. Exercise capacity in chronic fatigue syndrome. Arch Intern Med. 2000;160:3270-7.

24. Wallman KE, Morton AR, Goodman C, Grove R. Physiological responses during a submaximal cycle test in chronic fatigue syndrome. Med Sci Sports Exerc. 2004;36:1682-8.

25. Sisto SA, LaManca J, Cordero DL, Bergen MT, Ellis SP, Drastal S, et al. Metabolic and cardiovascular effects of a progressive exercise test in patients with chronic fatigue syndrome. Am J Med. 1996;100:634-40.

26. Fulcher KY, White PD. Strength and physiological response to exercise in patients with chronic fatigue syndrome. J Neurol Neurosurg Psychiatry. 2000;69:302-7.

27. Jammes Y, Steinberg JG, Mambrini O, Bregeon F, Delliaux S. Chronic fatigue syndrome: assessment of increased oxidative stress and altered muscle excitability in response to incremental exercise. $J$ Intern Med. 2005;257:299-310.

28. Sargent C, Scroop GC, Nemeth PM, Burnet RB, Buckley JD. Maximal oxygen uptake and lactate metabolism are normal in chronic fatigue syndrome. Med Sci Sports Exerc. 2002;34:51-6.

29. Vanness JM, Snell CR, Stevens SR. Diminished cardiopulmonary capacity during post-exertional malaise. Journal of Chronic Fatigue Syndrome. 2007;14:77-85.

30. Keller BA, Pryor JL, Giloteaux L. Inability of myalgic encephalomyelitis/chronic fatigue syndrome patients to reproduce VO(2)peak indicates functional impairment. J Transl Med. 2014;12:104.

31. Hodges LD, Nielsen T, Baken D. Physiological measures in participants with chronic fatigue syndrome, multiple sclerosis and healthy controls following repeated exercise: a pilot study. Clin Physiol Funct Imaging. 2018;38:639-44. 
32. Snell CR, Stevens SR, Davenport TE, Van Ness JM. Discriminative validity of metabolic and workload measurements to identify individuals with chronic fatigue syndrome. Phys Ther. 2013;93:1484-92.

33. Vermeulen RC, Kurk RM, Visser FC, Sluiter W, Scholte HR. Patients with chronic fatigue syndrome performed worse than controls in a controlled repeated exercise study despite a normal oxidative phosphorylation capacity. J Transl Med. 2010;8:93.

34. Faro M, Saez-Francas N, Castro-Marrero J, Aliste L, Fernandez de Sevilla T, Alegre J. Gender differences in chronic fatigue syndrome. Reumatol Clin. 2016;12:72-7.

35. Elmariah S, Goldberg LR, Allen MT, Kao A. Effects of gender on peak oxygen consumption and the timing of cardiac transplantation. J Am Coll Cardiol. 2006;47:2237-42.

36. Lai N, Martis A, Belfiori A, Tolentino-Silva F, Nasca MM, Strainic J, et al. Gender differences in V 02 and HR kinetics at the onset of moderate and heavy exercise intensity in adolescents. Physiol Rep. 2016;4:e12970.

37. Glaser S, Koch B, Ittermann T, Schaper C, Dorr M, Felix SB, et al. Influence of age, sex, body size, smoking, and beta blockade on key gas exchange exercise parameters in an adult population. Eur $\mathrm{J}$ Cardiovasc Prev Rehabil. 2010;17:469-76.

38. Wolfe F, Clauw DJ, Fitzcharles MA, Goldenberg DL, Katz RS, Mease P, et al. The American College of Rheumatology preliminary diagnostic criteria for fibromyalgia and measurement of symptom severity. Arthritis Care Res (Hoboken). 2010;62:600-10.

39. Martina JR, Westerhof BE, van Goudoever J, de Beaumont EM, Truijen J, Kim YS, et al. Noninvasive continuous arterial blood pressure monitoring with Nexfin(R). Anesthesiology. 2012;116:1092-103.

40. Viehoff PB, van Genderen FR, Wittink H. Upper limb lymphedema 27 (ULL27): Dutch translation and validation of an illness-specific health-related quality of life questionnaire for patients with upper limb lymphedema. Lymphology. 2008;41:131-8.

41. Mezzani A. Cardiopulmonary exercise testing: basics of methodology and measurements. Ann Am Thorac Soc. 2017;14(Supplement 1):3-11.

42. Dugan SA, Everson-Rose SA, Karavolos K, Sternfeld B, Wesley D, Powell LH. The impact of physical activity level on SF-36 role-physical and bodily pain indices in midlife women. J Phys Act Health. 2009;6:33-42.

43. McHorney CA, Ware JE Jr, Raczek AE. The MOS 36-Item Short-Form Health Survey (SF-36): II. Psychometric and clinical tests of validity in measuring physical and mental health constructs. Med Care. 1993;31:247-63.

44. Jason L, Brown M, Evans M, Anderson V, Lerch A, Brown A, et al. Measuring substantial reductions in functioning in patients with chronic fatigue syndrome. Disabil Rehabil. 2011;33:589-98.

45. Thorpe T, McManimen S, Gleason K, Stoothoff J, Newton JL, Strand EB, et al. Assessing current functioning as a measure of significant reduction in activity level. Fatigue. 2016;4:175-88.

46. Franklin JD, Atkinson G, Atkinson JM, Batterham AM. Peak Oxygen Uptake in Chronic Fatigue Syndrome/Myalgic Encephalomyelitis: A Meta-Analysis. Int J Sports Med. 2019;40(2):77-87. 
47. Ickmans K, Clarys P, Nijs J, Meeus M, Aerenhouts D, Zinzen E, et al. Association between cognitive performance, physical fitness, and physical activity level in women with chronic fatigue syndrome. $J$ Rehabil Res Dev. 2013;50:795-810.

48. Nijs J, De MK, Wolfs S, Duquet W. Disability evaluation in chronic fatigue syndrome: associations between exercise capacity and activity limitations/participation restrictions. Clin Rehabil. 2004;18:139-48.

49. Brown MM, Jason LA. Functioning in individuals with chronic fatigue syndrome: increased impairment with co-occurring multiple chemical sensitivity and fibromyalgia. Dyn Med. 2007;6:9.

50. Friedberg F. Does graded activity increase activity? A case study of chronic fatigue syndrome. J Behav Ther Exp Psychiatry. 2002;33:203-15.

51. Evering RM, Tonis TM, Vollenbroek-Hutten MM. Deviations in daily physical activity patterns in patients with the chronic fatigue syndrome: a case control study. J Psychosom Res. 2011;71:12935 .

52. Guazzi M, Adams V, Conraads V, Halle M, Mezzani A, Vanhees L, et al. EACPR/AHA Scientific Statement. Clinical recommendations for cardiopulmonary exercise testing data assessment in specific patient populations. Circulation. 2012;126:2261-74.

53. Brown AE, Jones DE, Walker M, Newton JL. Abnormalities of AMPK activation and glucose uptake in cultured skeletal muscle cells from individuals with chronic fatigue syndrome. PLoS One. 2015;10:e0122982.

54. Jones DE, Hollingsworth KG, Jakovljevic DG, Fattakhova G, Pairman J, Blamire AM, et al. Loss of capacity to recover from acidosis on repeat exercise in chronic fatigue syndrome: a case-control study. Eur J Clin Invest. 2012;42:186-94.

\section{Figures}



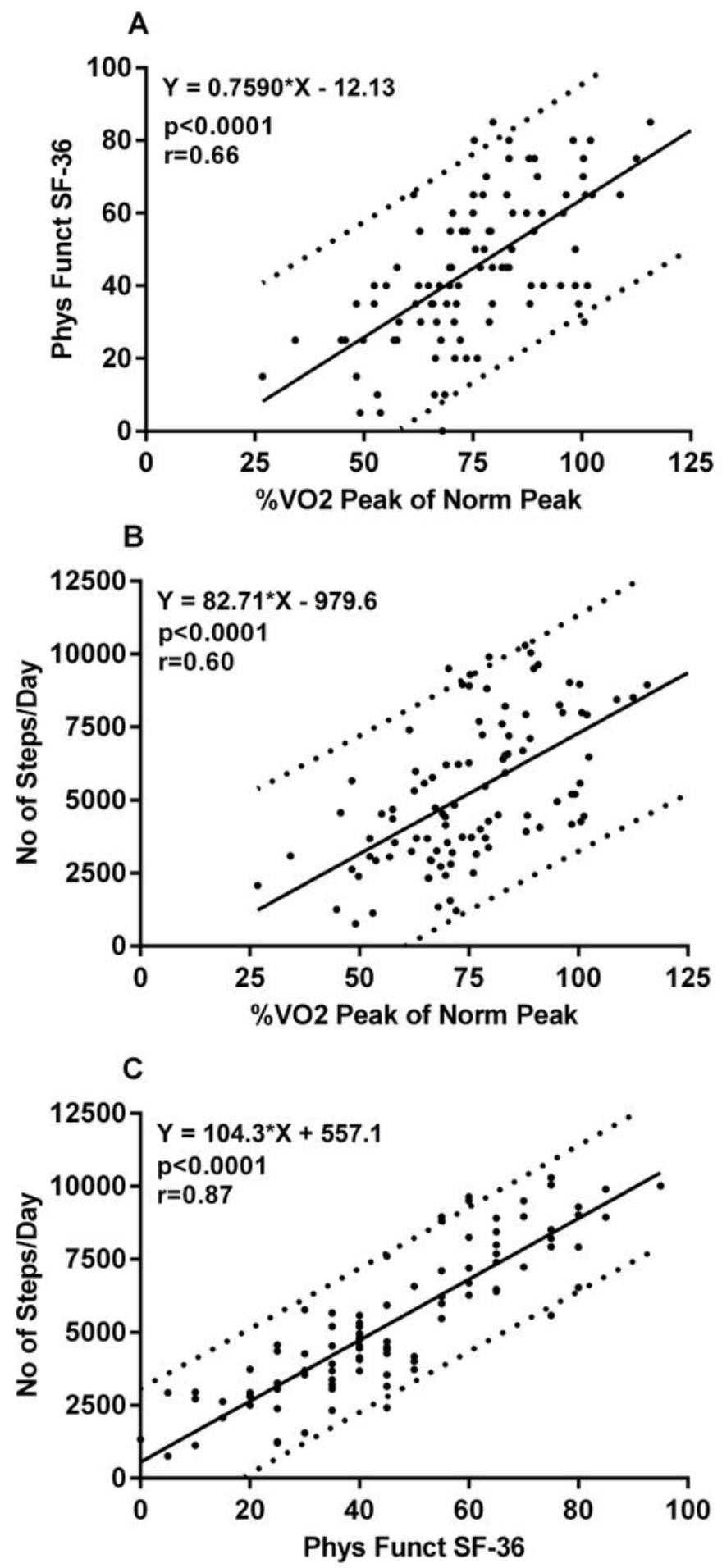

\section{Figure 1}

Correlations and $95 \%$ prediction intervals between the three measures of physical activity. Panel A shows the correlation between the SF-36 physical functioning scale and the percentage peak VO2 relative to controls [37]; panel B shows the correlation between the number of steps/day and the percentage peak VO2; panel $\mathrm{C}$ shows the correlation between the number of steps/day and the SF-36 physical functioning 
scale. All correlations were highly significant.Phys Funct SF-36: the physical functioning scale of the SF36 questionnaire; \%peak VO2 of Norm: peak VO2 oxygen uptake relative to controls.
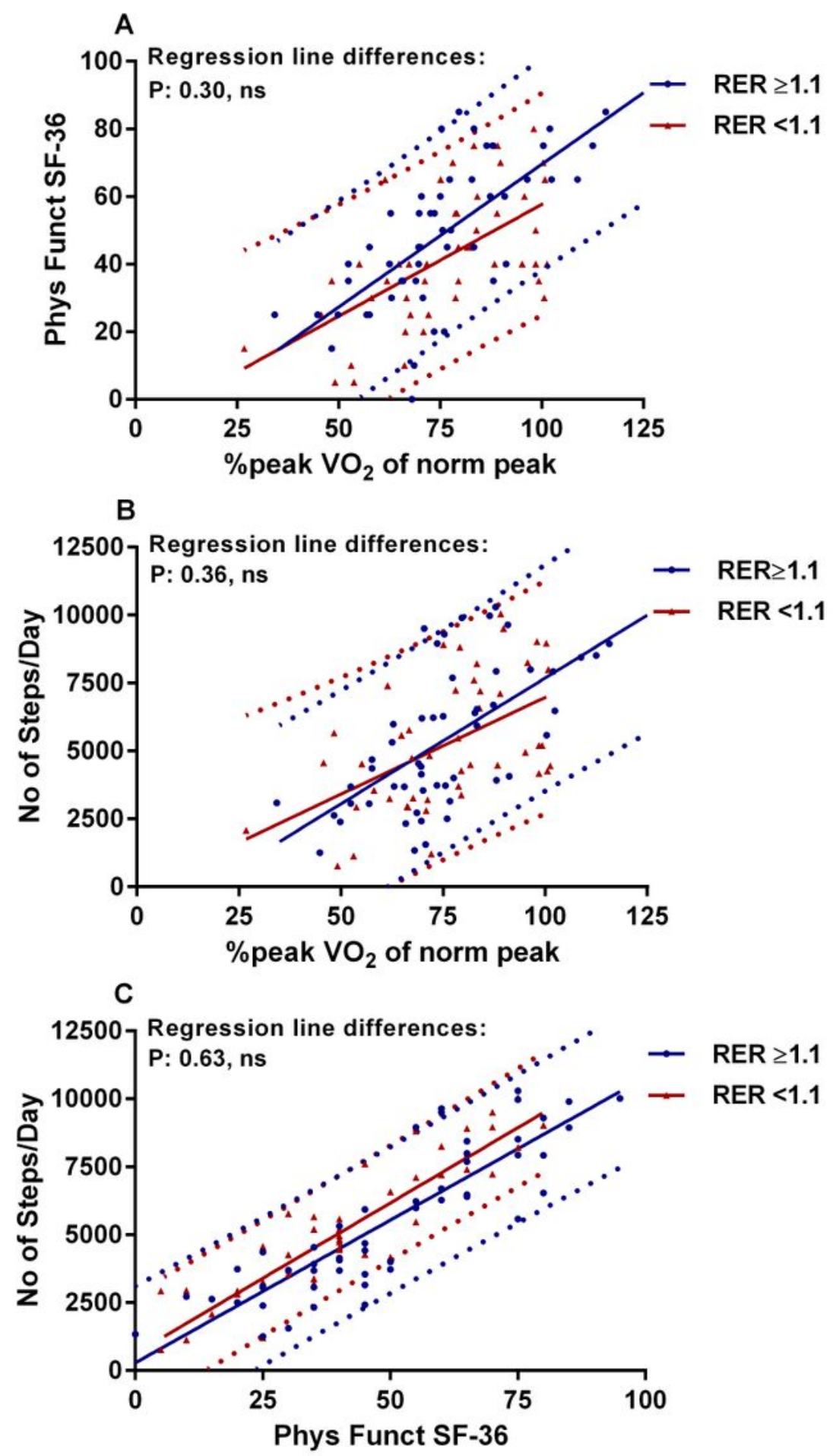

Figure 2

Correlations and $95 \%$ prediction intervals between the three measures in those with and without RER $\geq$ 1.1. Panel A shows the correlation between the SF-36 physical functioning scale and the percentage peak V02, panel B shows the correlation between the number of steps/day and the percentage peak VO2 and 
panel $\mathrm{C}$ shows the correlation between the number of steps/day and the SF-36 physical functioning scale. No significant differences were found in comparing RER<1.1 and RER $\geq 1$.1. Phys Funct SF-36: the physical functioning scale of the SF-36 questionnaire; \%peak VO2 of Norm: peak VO2 oxygen uptake relative to controls.
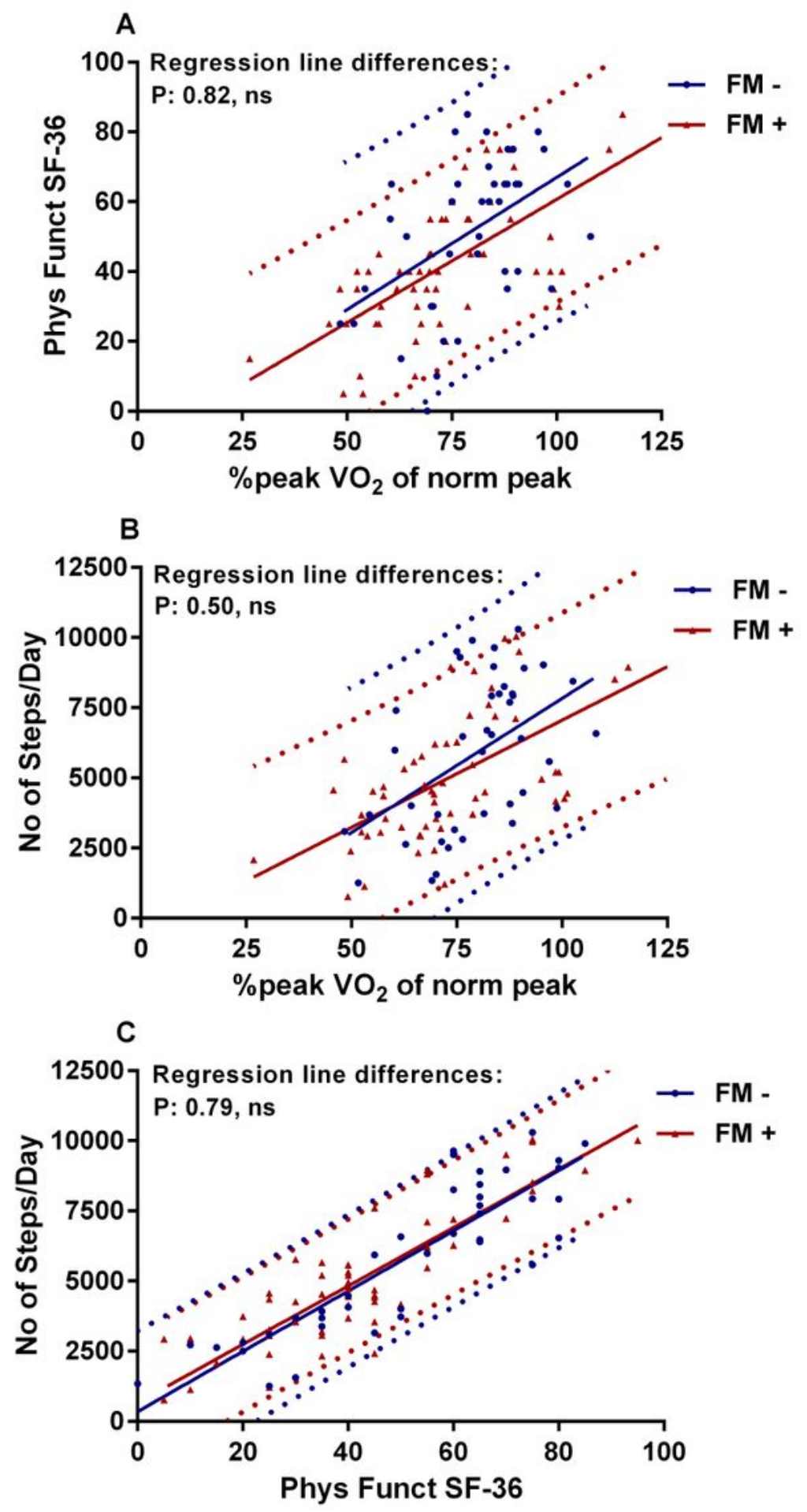

Figure 3 
Correlations and $95 \%$ prediction intervals between the three measures in those with and without fibromyalgia. Panel A shows the correlation between the SF-36 physical functioning scale and the percentage peak VO2, panel B shows the correlation between the number of steps/day and the percentage peak VO2 and panel C shows the correlation between the number of steps/day and the SF-36 physical functioning scale. No significant differences were found in comparing the absence or presence of fibromyalgia. Phys Funct SF-36: the physical functioning scale of the SF-36 questionnaire; \%peak VO2 of Norm: peak VO2 oxygen uptake relative to controls.

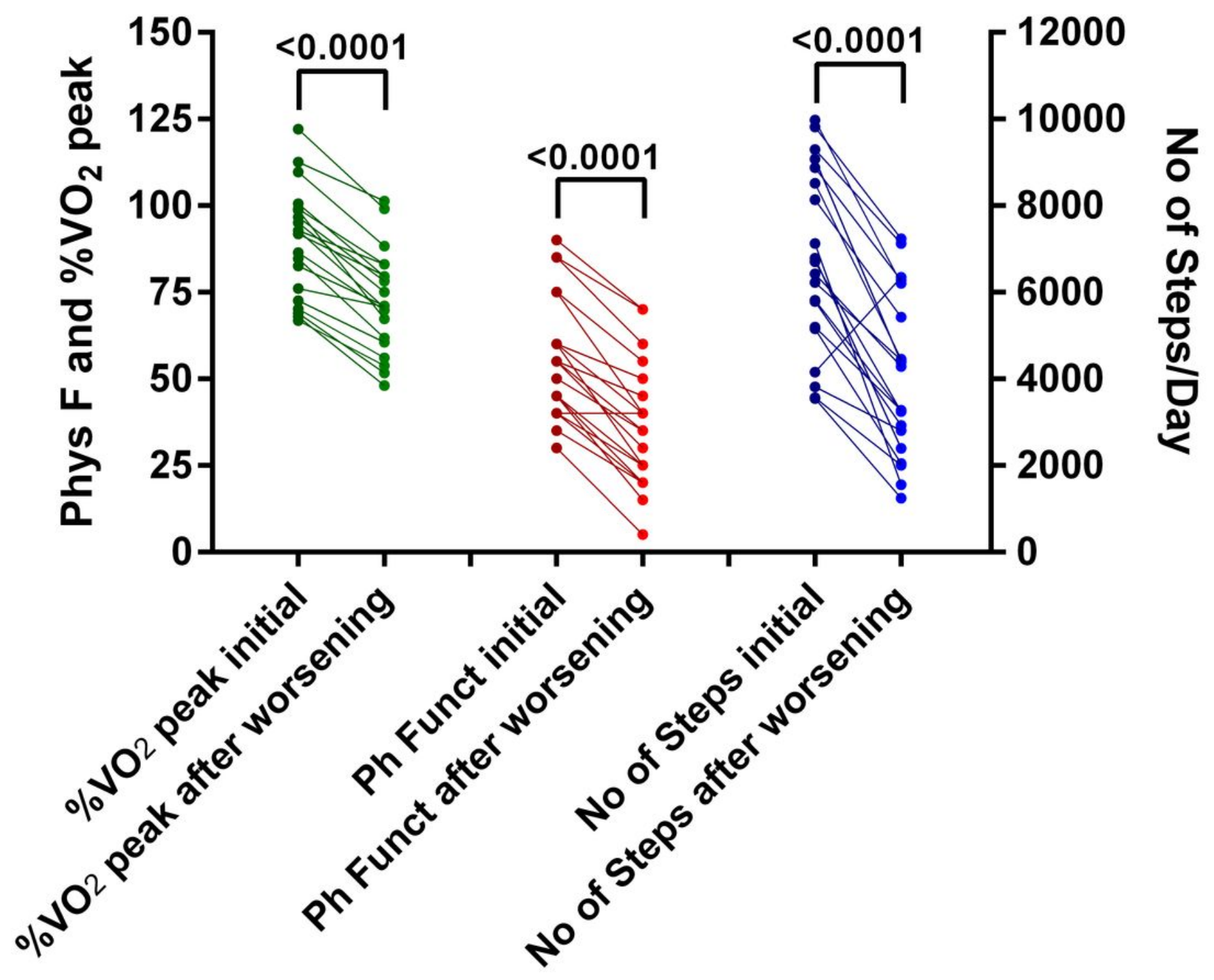

Figure 4

Changes in the three measures in 20 individuals re-tested after experiencing a clinical deterioration. Individual changes in the \%peak VO2 of normal, the physical functioning scale of the SF-36 questionnaire, and the number of steps/day in 20 patients undergoing re-evaluation because of worsening of symptoms. All mean measurements during the re-evaluation showed a significant 
deterioration $(P<.0001)$ in comparison to the initial evaluation. Phys $F=$ Physical Functioning scale of the SF-36 questionnaire; \%peak V02: peak VO2 oxygen uptake relative to controls [37]; No of Steps: number of steps per day. 